\section{The Presence of Ethanol in Radiopharmaceutical Injections}

TO THE EDITOR: As ethanol is frequently used in radiopharmaceutical injections, we were interested in the effect of ethanol by injection. Several questions crossed our mind. What is the maximum concentration of ethanol that we can inject without side effects? What is the hemolytic potential of ethanol? Can we dilute ethanol-containing injections just with water?

Ethanol can be used in radiopharmaceutical injections to enhance the solubility of highly lipophilic tracers or to decrease adsorption to vials, membrane filters, and injection syringes. More often, ethanol is present as a residual solvent from the eluent for elution of the radiopharmaceutical from a solid phase extraction cartridge or from the mobile phase used to purify the radiopharmaceutical by high performance liquid chromatography (HPLC). Using HPLC, ethanolbuffer mixtures have a relatively high viscosity that results in a high column back pressure, but ethanol has the advantage over other organic solvents of not necessarily requiring complete removal before intravenous injection of the diluted isolated HPLC fraction. After HPLC purification, ethanol can be removed from the collected fraction by evaporation, and the residue is then dissolved in or diluted with normal saline. However, this process delays the use of the radiopharmaceutical, increases the risk of radioactive contamination and the radiation dose to the manipulator, can be accompanied by partial decomposition of the tracer, and may result in adsorption of highly lipophilic tracers to the filter membrane used for sterile filtration and to the vial, rubber stopper, and syringes. An alternative is not to remove the ethanol but to dilute the isolated HPLC fraction to an acceptably low ethanol concentration. The European Medicines Agency and the U.S. Food and Drug Administration have noted that higher amounts of ethanol (more than $0.5 \%$, the common limit on class 3 residual solvents) may be acceptable provided they are realistic in relation to manufacturing capability and good manufacturing practice (1). Indeed, several solutions of poorly soluble nonradioactive pharmaceutical compounds for injection contain ethanol as a cosolvent in concentrations of up to $20 \%$ (2). However, to safely use ethanol in radiopharmaceutical injections, one must address several questions.

Potential side effects of ethanol on injection are pain and hemolysis, depending on the concentration. To reduce the risk of pain and hemolysis, ethanol-containing formulations should be injected slowly. This will decrease the concentration at the site of injection because of rapid mixing with large volumes of blood. Depending on the concentration, ethanol also causes hemolyses by forming membrane pores in red blood cells by which the cells finally swell and burst through a colloid-osmotic mechanism. At a low concentration, ethanol protects red blood cells from hypotonic hemolysis by partitioning into the cell membrane to increase its surface area. The osmolality of ethanol is calculated to be 2,029 $\mathrm{mOsm} / \mathrm{kg}$, which means that a mixture of $1.39 \%$ ethanol in water is isotonic. A solution for injection containing $10 \%$ ethanol, a limit used in several radiopharmaceutical centers, thus would be hypertonic on a theoretic basis. However, ethanol should not be considered in calculations of osmolality as it freely diffuses through the cell membrane. Solutions of ethanol in $0.9 \% \mathrm{NaCl}$ show no hemolysis, whereas dilution of blood with an ethanol-water $(7: 93 \mathrm{v} / \mathrm{v})$ mixture induces massive hemolysis. HPLC fractions isolated during purification of radiopharmaceuticals should thus be diluted with normal saline and not with water, as the latter situation would result in a hypotonic solution and cause hemolysis. $\mathrm{NaCl}$ solutions are isotonic at $0.9 \%$ (normal saline), but red blood cells are resistant to lysis in hypertonic solutions of $\mathrm{NaCl}$ up to $3.6 \%$. Many cosolvents show drastic changes in hemolytic potential as the concentration of $\mathrm{NaCl}$ in the aqueous component increases, but the hemolytic potential of ethanol is fairly constant when mixed with $0.9 \%-2 \%$ $\mathrm{NaCl}$ solutions (3).

Injections of radiopharmaceutical preparations rarely exceed $20 \mathrm{~mL}$. For a $10 \%$ ethanol solution, which is frequently used and generally accepted, a 20-mL injection would mean the administration of $2 \mathrm{~mL}$ of ethanol, resulting in a temporary and rapidly decreasing blood concentration of 0.44 per mille (assuming a blood volume of $4.7 \mathrm{~L}$ for a $70-\mathrm{kg}$ adult). This is below the $0.5 \%$ driver's limit in force in many European countries. Special attention should be paid to injections for children, women during pregnancy and lactation, persons with alcoholism, and high-risk groups such as persons with liver disease or epilepsy.

For some preparations such as ${ }^{18} \mathrm{~F}-\mathrm{FDG}$, the presence of ethanol has been shown to reduce radiolysis, which may be an additional advantage of including ethanol in the formulation of radiopharmaceuticals (4).

In conclusion, ethanol is the preferred organic solvent in the mobile phases used for HPLC purification of short-lived radiopharmaceuticals, as isolated HPLC fractions can safely be used without removal of the organic solvent after appropriate dilution with normal saline to a concentration of ethanol not exceeding $10 \%$ and an injection volume below $20 \mathrm{~mL}$.

\section{REFERENCES}

1. Guidance for industry on Q3C impurities: residual solvents (CPMP/ICH/283/95). Available at: http://www.emea.europa.eu/pdfs/human/ich/028395en.pdf or http:// www.fda.gov/cber/gdlns/q3cresolvent.pdf. Accessed September 17, 2008.

2. Strickley RG. Solubilizing excipients in oral and injectable formulations. Pharm Res. 2004;21:201-230.

3. Reed KW, Yalkowsky SH. Lysis of human red blood cells in the presence of various cosolvents. III. The relationship between hemolytic potential and structure. J Parenter Sci Technol. 1987;41:37-39.

4. Kiselev MY, Tadino V. Eastern Isotopes, Inc. Stabilization of radiopharmaceuticals labeled with 18-F. W.O. 2004/043497 A1. May 27, 2004.

Kim Serdons
Alfons Verbruggen
Guy Bormans
K.U. Leuven
Leuven, Belgium

DOI: 10.2967/jnumed.108.057026 